\title{
FIRST-PASSAGE TIME OF MARKOV PROCESSES TO MOVING BARRIERS
}

\author{
HENRY C. TUCKWELL,* Monash University \\ FREDERIC Y. M. WAN, ** University of British Columbia
}

\begin{abstract}
The first-passage time of a Markov process to a moving barrier is considered as a first-exit time for a vector whose components include the process and the barrier. Thus when the barrier is itself a solution of a differential equation, the theory of first-exit times for multidimensional processes may be used to obtain differential equations for the moments and density of the first-passage time of the process to the barrier. The procedure is first illustrated for first-passage-time problems where the solutions are known. The mean first-passage time of an Ornstein-Uhlenbeck process to an exponentially decaying barrier is then found by numerical solution of a partial differential equation. Extensions of the method to problems involving Markov processes with discontinuous sample paths and to cases where the process is confined between two moving barriers are also discussed.
\end{abstract}

EXIT TIMES; DIFFUSION PROCESS; NEURAL FIRING; ORNSTEIN-UHLENBECK PROCESS

\section{Introduction}

The problem of determining the first-passage times to a moving barrier for diffusion and other Markov processes arises in biological modeling, in statistics and in engineering. In population genetics (see Ewens (1979)), if $X(t)$ is the number of a certain kind of genes present at time $t$ in a population with a total of $\mathrm{N}(\mathrm{t})$ genes, then the time at which $\mathrm{X}(\mathrm{t})$ first hits $\mathrm{N}(\mathrm{t})$ is the time of fixation of that gene in the population. In neurophysiology (Holden (1976)), if $X(t)$ is the displacement of a nerve-cell voltage from its resting level and $\boldsymbol{\theta}(\boldsymbol{t})$ is the threshold voltage displacement, then the time at which $\mathrm{X}(\mathrm{t})$ first hits $\boldsymbol{\theta}(\boldsymbol{t})$ is the time at which an action potential is generated. In statistics the problem of determining the time of first passage of a Wiener process to certain moving

Received 12 July 1983.

* Postal address: Department of Mathematics, Monash University, Clayton, VIG168, Australia.

** Present address: Applied Mathematics Program, FS-20, University of Washington, Seattle, WA 98195, USA.

Research partly supported by NSERC of Canada Operating-Grant No. A9259 and by U.S. NSF Grant No. MCS-8306592. 
barriers arises asymptotically in sequential analysis (Darling and Siegert (1953)) and in computing the power of statistical tests (Durbin (1971)). A review of applications in engineering can be found in Blake and Lindsey (1973). Only in the case of the time of first passage of a Wiener process to a linear barrier is a closed-form expression for the density available (see, for example, Cox and Miller (1965)). Approximate or numerical methods have been employed for other boundary types (Ferebee (1982)).

In the first part of this paper we obtain partial differential equations for the moments of the first-passage time of a diffusion process to a general class of moving barriers. Some examples will be given for which exact solutions of such equations are obtained by means of transformations to simpler problems with a known solution. We also consider a first-passage-time problem for which useful new results can be obtained by numerical solutions of the moment equations. Extensions of the method of solution to finding the moments and density of the first-passage time of continuous and discontinuous Markov processes confined between two moving barriers will also be discussed.

\section{The moments of first-passage time}

Let $\{X(t), t \geqq 0\}, X(O)=x$, be a temporally homogeneous diffusion process with Ito stochastic differential

$$
d X=\alpha(X) d t+\beta(X) d W,
$$

where $\{\mathrm{W}(\mathrm{t}), t \geqq 0\}$ is a standard Wiener process with zero mean and variance $\mathrm{t}$. We assume that existence and uniqueness conditions (see Gihman and Skorohod (1972)) are met. Let $\{\mathrm{Y}(\mathrm{t}), t \geqq 0\}$ be a moving barrier, with $\mathrm{Y}(\mathrm{O})=\mathrm{y}$. We shall take $\mathrm{X}(\mathrm{O})<\mathrm{Y}(\mathrm{O})$, as it is more natural in most settings. (The case $\mathrm{X}(\mathrm{O})>\mathrm{Y}(\mathrm{O})$ is handled throughout by reversing the inequality of the form $\mathrm{x}<\mathrm{y}$ and by making suitable adjustments to certain boundary conditions.)

The random variable $\mathrm{T}(\mathrm{x}, \mathrm{y})$ is defined as the time at which $\mathrm{X}$ first hits the moving barrier $\mathrm{Y}$ :

$$
T(x, y)=\inf \{\mathrm{t}|X(t)=Y(t)| X(O)=x<y=Y(O)\} .
$$

The underlying mechanisms are sketched in Figure 1. It is further assumed that $T$ is a 'proper' random variable, in the sense that

$$
\operatorname{Pr}[T<\infty]=1 \text {, }
$$

and has finite moments of order $\boldsymbol{n} \leqq \boldsymbol{n}_{\mathbf{0}}$;

$$
M_{n}(x, y)=E\left[T^{n}(x, y)\right], \quad n \leqq n_{0} .
$$

Our method for determining $\boldsymbol{M}_{n}(\boldsymbol{x}, \mathrm{y})$ requires that $Y(t)$ be a component $Y_{1}(t)$, say, of a vector function $Y(t) \equiv\left(Y_{1}(t), \cdot, Y_{k}(t)\right)$ which satisfies a 


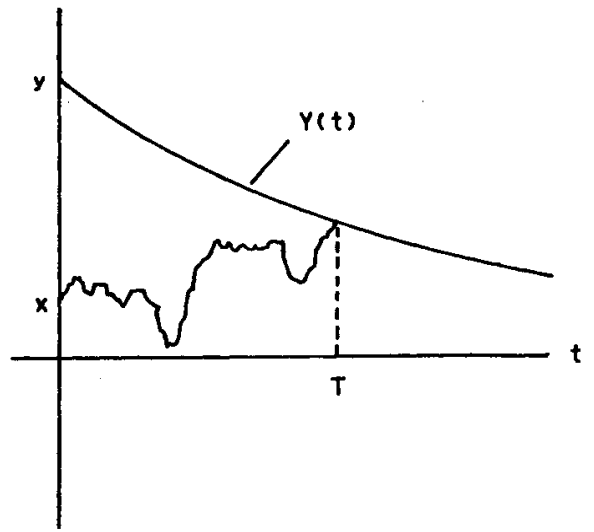

Figure 1. The random process hits the moving barrier $Y(t)$, the time of first passage being marked $T$.

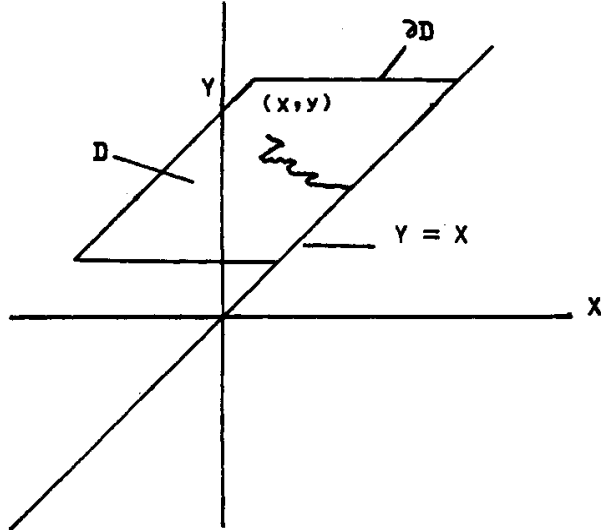

Figure 2. The trajectories in Figure 1 as they appear in the (x, y)-plane. $D$ is a domain enclosing the initial point $(\mathrm{x}, \mathrm{y})$ and its boundary is $\partial D$.

first-order vector differential equation of the form

$$
\frac{d Y}{d t}=\gamma(Y) \quad(t>0), \quad Y(0)=y .
$$

(If $\mathrm{Y}$ is the solution of a non-autonomous system, then $\mathrm{Y}^{*} \equiv$ $\left(Y_{1}(t)\right.$, । $\left.7 Y_{k}(t), Y_{k+1}(t)\right)$ with $d Y_{k+1} / d t=1$ is the solution of an augmented autonomous system.)

In what follows, we limit our discussion first to the case where (2.5) is a scalar ordinary differential equation (ORE),

$$
\frac{d Y}{d t}=\gamma(Y) \quad(t>0), \quad Y(0)=y .
$$

With (2.6), we may now consider the vector random process $(X, Y)$ which satisfies the degenerate system of first-order Ito stochastic equations

$$
d\left(\begin{array}{l}
X \\
Y
\end{array}\right)=\left(\begin{array}{l}
\alpha(X) \\
\gamma(Y)
\end{array}\right) d t+\left[\begin{array}{cc}
\beta(X) & 0 \\
0 & 0
\end{array}\right] d\left(\begin{array}{l}
W_{1} \\
W_{2}
\end{array}\right),\left(\begin{array}{l}
X(0) \\
Y(0)
\end{array}\right)=\left(\begin{array}{l}
x \\
y
\end{array}\right)
$$

and treat the first-passage-time problem for $\mathrm{X}$ as a first-exit-time problem for the vector $(\mathrm{X}, \mathrm{Y})$ in the plane. The separate trajectories of $X(t)$ and $Y(t)$ sketched in Figure 1, appear as in Figure 2 when plotted in the $X$, Y-plane. We therefore see that the time of first passage of $\mathrm{X}$ to $\mathrm{Y}$ is the time of first exit of $(\mathrm{X}, \mathrm{Y})$ from all or some part of the half-plane, $\boldsymbol{x}<\mathrm{y}$. The theory of first-exit times for multidimensional diffusion processes (Dynkin (1965); Gihman and Skorohod (1972)) thus enables us to obtain differential equations for the moments, $M_{n}(x, y)$. 
If $\mathrm{P}$ is the transition probability function of $(\mathrm{X}, \mathrm{Y})$ so that

$$
P(t, x, y ; \bar{t}, \bar{x}, \bar{y})=\operatorname{Pr}[X(\bar{t}) \leqq \bar{x}, Y(\bar{t}) \leqq \bar{y} \mid X(t)=x, Y(t)=y]
$$

then $\mathbf{P}$ satisfies the backward Kolmogorov equation

$$
-\frac{\partial P}{\partial t}=\frac{1}{2} \beta^{2}(x) \frac{\partial^{2} P}{\partial x^{2}}+\alpha(x) \frac{\partial P}{\partial x}+\gamma(y) \frac{\partial P}{\partial y}=\mathscr{L}_{x y}(P) .
$$

(Since $\mathrm{X}$ and $\mathrm{Y}$ are independent, $\mathrm{P}$ can actually be written as the product of transition probabilities for $\mathrm{X}$ and $\mathrm{Y}$ but we ignore this here.)

Furthermore, the moments of the first-exit time satisfy the recursion system of equations

$$
\mathscr{L}_{x y}\left(M_{n}\right)=-n M_{n-1}, \quad(x, y) \in D,
$$

where $\boldsymbol{D}$ is a set of points $(\boldsymbol{x}, \mathrm{y})$ from which passage of $(\mathrm{X}(\mathrm{t}), \mathrm{Y}(\mathrm{t}))$ to the line $\mathrm{x}=\mathrm{y}$ is certain. These equations may be solved to obtain the moments of the time of first passage of the scalar diffusion $\mathrm{X}$ to the moving barrier $\mathrm{Y}$.

In order to solve Equation (2.10) for the moments of the first-passage time, boundary conditions must be specified. Let $\boldsymbol{D}$ be a set of points in the (x, y)-plane with boundary $\partial D$, such as that sketched in Figure 2. That is, part of $\partial D$ is along $\mathrm{y}=\mathrm{x}$. Assuming that escape from $\boldsymbol{D}$ is certain for $(\mathrm{X}, \mathrm{Y})$, the boundary conditions are

$$
M_{n}(x, y)=0, \quad(x, y) \in \partial D,
$$

since if $(\mathrm{x}, \mathrm{y}) \in \partial \boldsymbol{D}, \boldsymbol{T}(\boldsymbol{x}, \boldsymbol{y})=\boldsymbol{0}$ with probability 1 .

In cases where escape of $(\mathrm{X}, \mathrm{Y})$ from the half-plane $\boldsymbol{H}=\{(\mathrm{x}, \mathrm{y}) \mid \boldsymbol{x}<\boldsymbol{y}\}$ is certain, one may first find results for finite $\boldsymbol{D}$ and then take limits as $\boldsymbol{D}$ increases to cover $\boldsymbol{H}$ (cf. Cox and Miller (1965)). In problems where numerical solution of the moment equations is necessary, the region $\boldsymbol{D}$ is chosen such that points (x,y) at which the solution is required are away from $\boldsymbol{\partial D}$. The size of $\boldsymbol{D}$ is then increased until further increases lead to a change which is less than a small specified change in the solution at the points of interest. This procedure will be illustrated in the next section.

As the actual problem has only a one-sided exit, a reflecting barrier along $\partial D$ except for the portion along $\mathrm{x}=\mathrm{y}$ (denoted by $\partial D^{\prime}$ ) seems just as appropriate as an absorbing barrier. In other words, instead of $\boldsymbol{M}_{\boldsymbol{n}}=\boldsymbol{0}$, we could have

$$
\frac{\partial M_{n}}{\partial \nu}=0, \quad(x, y) \in \partial D^{\prime}
$$

where $\partial() / \partial \nu$ is the derivative of $(\cdot)$ normal to $\partial D^{\prime}$. Numerical calculations done on the firing-time problem for a model neuron (in Section 4 of this paper and in Tuckwell et al. (1984)) indicate that both types of boundary conditions 
along $\partial D^{\prime}$ give effectively the same $M_{n}(x, y)$ for (x, y) away from the boundary provided that the finite domain $\mathrm{D}$ is sufficiently large. That is, for points remote from $\partial D, M_{n}(\mathbf{x}, \mathbf{y})$ is insensitive to the choice of the supplementary boundary condition on $\partial D^{\prime}$. The condition (2.12) has the advantage that it eliminates the appearance of a boundary-layer phenomenon in the solution adjacent to $\partial D^{\prime}$ sometimes associated with the Dirichlet problem. However we note from the exact solutions of Section 3 that the limiting solutions, obtained by first applying either a reflecting or an absorbing barrier condition on $\partial D^{\prime}$, do not themselves satisfy those conditions at $\infty$.

\section{Some exact solutions}

To illustrate the application of the method outlined in the last section, we consider here three, albeit simple, examples for which the first-passage-time moments can be obtained exactly.

(i) Wiener process with drift - constant barrier. The density and moments of the time of first passage of a Wiener process with drift to a constant barrier are well known. The theory outlined above approaches this problem from a different viewpoint. The stochastic differential for the diffusion is

$$
d X=\mu d t+\sigma d W, \quad X(0)=x,
$$

and the barrier equation is

$$
\frac{d Y}{d t}=0 ! \quad Y(0)=y .
$$

Thus, we have $\alpha=\mu, \beta=\sigma$ and $\gamma \equiv 0$. With $x=\mathrm{X}(\mathrm{O})<\mathrm{Y}(\mathrm{O})=y$, the moments $M_{n}(x, y)$ of the time of first passage of $\mathrm{X}$ to $\mathrm{Y}$ satisfy

$$
\frac{1}{2} \sigma^{2} \frac{\partial^{2} M_{n}}{\partial x^{2}}+\mu \frac{\partial M_{n}}{\partial x}=-n M_{n-1}, \quad(x<y),
$$

$n=1,2, \cdots$, with boundary condition $M_{n}(y, y)=0$.

The equations for the moments (3.3) for a constant barrier are evidently ODE in the independent variable $\boldsymbol{x}$ with $\mathrm{y}$ appearing as a parameter. By the method of Section 2, we first solve the oDE on the strip y $-\mathbf{a}<x<y$, with its solution denoted by $\boldsymbol{M}_{n}^{(\alpha)}(\boldsymbol{x}, \mathbf{y})$, and with the boundary conditions $\boldsymbol{M}_{n}^{(a)}(y, \mathbf{y})=$ $M_{n}^{(a)}(y-a, y)=0$. The moments $\left.\boldsymbol{M}_{n}(\boldsymbol{x}, \mathbf{y})(\mathrm{n}=1,2,)^{\prime}\right)$, are obtained by letting $\mathrm{a} \rightarrow \infty$. The solution for $\boldsymbol{M}_{1}^{(a)}(\boldsymbol{x}, \mathrm{y})$ is

$$
M_{1}^{(a)}(x, y)=\frac{\mathrm{v}-x}{\mu} \frac{\mathrm{a}}{\mu} \frac{1-\exp \left(-2 \mu(x-y) / \sigma^{2}\right)}{1-\exp \left(2 \mu a / \sigma^{2}\right)}
$$

For $\boldsymbol{\mu}>\mathbf{0}$, we get in the limit as $\mathbf{a} \rightarrow \infty$

$$
M_{1}(x, y)=\frac{y-x}{\mu}
$$


Similarly, we find

$$
M_{2}(x, y)=\frac{\sigma^{2}(y-x)}{\mu^{3}}+\frac{(\mathrm{y}-x)^{2}}{\mu^{2}} .
$$

The above expression for $\boldsymbol{M}_{1}$ and $\boldsymbol{M}_{2}$ thus agree with the known results (Cox and Miller (1965)).

It should be noted that the same limiting solutions for $M_{1}$ and $M_{2}$ would also be obtained had we used $\left[\partial M_{n}^{(a)} / \partial x\right]_{x=y-a}=0$ instead of $M_{n}^{(a)}(y-a, y)=0$ before taking the limit $a \rightarrow \infty$.

(ii) Wiener process with drift - linear barrier. For this case, the equation for $\mathrm{X}$ is as in the previous example but the barrier $\mathrm{Y}(\mathrm{t})$ now satisfies

$$
\frac{d Y}{d t}=k, \quad Y(0)=\mathrm{y} .
$$

To obtain the moments of the first-passage time of $X(t)$ to $Y(t)$ with $x=\mathrm{X}(\mathrm{O})<$ $\mathrm{Y}(\mathrm{O})=y$, it is simplest to set $X(t)=\hat{X}(t)+k t$ and write (3.1) as

$$
d \hat{X}=(\mu-k) d t+\sigma d W, \quad \hat{X}(0)=x .
$$

Then the original first-passage-time problem becomes that of $\hat{X}(t)$ to a constant barrier $\mathrm{y}$ and the solution given in (i) is applicable. With $\boldsymbol{\alpha}=\boldsymbol{\mu}-k, \boldsymbol{\beta}=\boldsymbol{\sigma}$ and $\gamma \equiv 0$, we have from (3.5) and (3.6)

$$
M_{1}(x, y)=\frac{y-x}{\mu-k}, \quad M_{2}(x, y)=\frac{\left(\sigma^{2}(y+x)\right.}{(\mu-k)^{3}}+\frac{\bar{y} x)^{2}}{(\mu-k)^{2}}
$$

which coincide with known results (Cox and Miller (1965)).

To obtain the same solution directly by the method of Section 2, without the transformation from $X$ to $\hat{X}$, we note that with $\alpha=\mu, \beta=\sigma$ and $\gamma \equiv k$, the moments of the first-passage time of $X(t)$ to $Y(t)$ satisfy

$$
\frac{1}{2} \sigma^{2} \frac{\partial^{2} M_{n}}{\partial x^{2}}+\mu \frac{\partial M_{n}}{\partial x}+k \frac{\partial M_{n}}{\partial y}=-n M_{n-1}, \quad(x<y) .
$$

$n=1,2, \cdots$, with $M_{n}(y, y)=0$. The equation may be transformed by a change of variables $\xi=x-y$ and $\boldsymbol{\eta}=\boldsymbol{x}+\mathrm{y}$ into

$$
\frac{\partial^{2} M_{n}}{\partial \xi^{2}}+2 \frac{\partial^{2} M_{n}}{\partial \xi \partial \eta}+\frac{\partial^{2} M_{n}}{\partial \eta^{2}}+2 \mu_{d} \frac{\partial M_{n}}{\partial \xi}+2 \mu_{s} \frac{\partial M_{n}}{\partial \eta}=-\frac{2}{\sigma^{2}} n M_{n-1}
$$

with $M_{0} \equiv 1$ and with

$$
\mu_{s}=\frac{\mu+k}{\sigma^{2}}, \quad \mu_{d}=\frac{\mu}{\sigma^{2}}
$$

The solution domain is now the half-plane $\xi<0$ with the boundary condition $\boldsymbol{M}_{\boldsymbol{n}}(y, y)=O$ taking the form 


$$
M_{n}(\xi=0, \eta)=0 .
$$

The method of Section 2 solves (3.11) on a finite rectangle, say $\{-\boldsymbol{a}<\boldsymbol{\xi}<0$, $|\eta|<l\}$, and then lets $\mathbf{a}$ and $l$ in the solution tend to $\infty$.

For reasons which will become clear presently, we give here only a formal solution for $M_{1}^{(a)}(\xi, \eta)$ in the strip $\{-\mathbf{a}<\xi<0,|\eta|<\infty\}$ obtained by the method of generalized Fourier transform in $\eta$ :

$$
M_{1}^{(a)}(\xi, \eta)=\frac{a}{\mu-k}\left\{\exp \left(-\mu_{d}(\xi+a)\right) \frac{\sinh \left(\mu_{d} \xi\right)}{\sinh \left(\mu_{d} a\right)}-\frac{\xi}{a}\right\} .
$$

As $\mathbf{a} \rightarrow \infty$, we get (as previously found in (3.9)),

$$
M_{1}^{(a)}(\xi, \eta) \rightarrow M_{1}(\xi, \eta)=-\frac{\xi}{\mu-k}=\frac{y-x}{\mu-k} .
$$

Observe that the solution (3.14), which satisfies the differential equation (3.11), the boundary condition $M_{1}^{(a)}(0, \eta)=0$ and the auxiliary condition $M_{1}^{(a)}(-\mathrm{a}, \eta)=$ 0 , is independent of $\boldsymbol{\eta}$. As such $\boldsymbol{M}_{1}^{(\boldsymbol{a})}$ does not tend to 0 as $\boldsymbol{\eta} \rightarrow \pm \infty$. Instead, we have $\partial M_{1}^{(a)} / \partial \eta=0$ which suggests that a homogeneous Neumann condition may be more appropriate for the finite-domain problem in this case.

(iii) Gene fixation in a varying population. In a model of fluctuating gene frequency when selection varies randomly (Crow and Kimura (1970); Tuckwell (1976)), the number of genes of a certain type is described by a Malthusian law in the form of a Stratonovich differential:

$$
\mathrm{dX}=, \mathrm{uXdt}+\sigma X d W, \quad \mathrm{X}(0)=\mathrm{x} .
$$

As an Ito differential, (3.16) becomes (Jaswinski (1970)):

$$
\mathrm{dX}=\left(\mu+\frac{\sigma^{2}}{2}\right) \mathrm{Xdt}+\sigma X d W, \quad \mathrm{X}(0)=\mathrm{x} .
$$

The total number of genes in the population (at a particular locus) is assumed to grow according to the Malthusian law,

$$
\frac{d Y}{\mathrm{dt}}=k Y, \quad Y(O)=y .
$$

The gene frequency is thus $\mathrm{X} / \mathrm{Y}$ and when this reaches unity, or equivalently when $\mathrm{X}$ hits $\mathrm{Y}$, there will be only one kind of gene in the population (fixation). In this model it is necessary to have $\mathrm{X}(\mathrm{O}) \leqq \mathrm{Y}(\mathrm{O})$.

From the results of Section 2 we see that the moments of the fixation time are solutions of the equations

$$
\frac{\sigma^{2}}{2} x^{2} \frac{\partial^{2} M_{n}}{\partial x^{2}}+\left(\mu+\frac{\sigma^{2}}{2}\right) x \frac{\partial M_{n}}{\partial x}+k y \frac{\partial M_{n}}{\partial y}=-n M_{n-1}, \quad(x<y)
$$


$\mathrm{n}=1,2, \ldots$, with $M_{n}(y, \mathrm{y})=0$. For a solution of the problem (for $\boldsymbol{x}>0$ and $\mathrm{y}>0$ ), we set $\boldsymbol{\xi}=\operatorname{In}(\mathrm{x})$ and $\boldsymbol{\eta}=\operatorname{In}(\mathrm{y})$ and transform (3.19) into an equation of constant coefficients

$$
\frac{\sigma^{2}}{2} \frac{\partial^{2} M_{n}}{\partial \xi^{2}}+\mu \frac{\partial M_{n}}{\partial \xi}+k \frac{\partial M_{n}}{\partial \eta}=-n M_{n-1} \quad(\xi<\eta)
$$

with $M_{n}(\xi=\eta, \eta)=0$. For $\mu>k$, this is exactly the problem solved in (ii) and the solution there is immediately applicable.

In terms of $\boldsymbol{x}$ and $\boldsymbol{y}$, we have

$$
\begin{gathered}
M_{1}=\frac{\eta-\xi}{\mu-k}=\frac{\ln (y / x)}{\mu-k} \\
M_{2}=\frac{\sigma^{2}(\eta-\xi)}{(\mu-k)^{3}}+\frac{(\eta-\xi)^{2}}{(\mu-k)^{2}}=\frac{\sigma^{2} \ln (y / x)}{(\mu-k)^{3}}+\frac{[\ln (y / x)]^{2}}{(\mu-k)^{2}} .
\end{gathered}
$$

The results (3.21) and (3.22) can be obtained by transforming the system of equations for $\mathrm{X}$ and $\mathrm{Y}$ to that of a Wiener process with drift and a linear barrier (Tuckwell (1974)).

\section{Firing time of a model neuron}

The displacement $\mathrm{X}(\mathrm{t})$ of a nerve cell's electrical potential from its resting value has been represented, under certain conditions, by an OrnsteinUhlenbeck process. The stochastic differential is

$$
d X=(\alpha-X) d t+\beta d W, \quad X(0)=x .
$$

Here time is measured in units of the membrane time constant. When $X(t)$ reaches a threshold value $Y(t)$ the neuron fires an action potential. Usually one is interested in the case of an initially resting cell so that $\boldsymbol{x}=0$.

This diffusion approximation to the underlying discontinuous process in the model of Stein (1965) was first considered in the context of neural firing by Gluss (1967), and the mean first-passage time in the case of a constant Y was obtained by Roy and Smith (1969). The assumption of a constant threshold may not be appropriate, especially for a rapidly firing nerve cell. Usually, after a short time interval, called the absolutely refractory period, in which generation of a subsequent action potential is impossible, the threshold declines as the inhibitory effect of the previous action potential dies away.

Several time-dependent threshold functions $Y(t)$ have been proposed, both monotonic (Holden (1976), Chapter 4) and oscillatory (Wilbur and Rinzel (1983)). To illustrate the present method we consider in this section a generalization of the exponentially decaying threshold proposed by Weiss (1964). Let Y(t) satisfy 


$$
\frac{d Y}{d t}=-k_{1} Y+k_{2} \quad(t>0), \quad Y(0)=y
$$

with $k_{1}, k_{2} \geq \Omega$.The solution of the above initial-value problem (4.2) is

$$
Y(t)=\left(y-k_{2} / k_{1}\right) \exp \left(-k_{1} t\right)+k_{2} / k_{1} .
$$

For very small positive values of $\boldsymbol{k}_{1}, Y(t)$ will appear like a constant threshold with magnitude y provided $\boldsymbol{\alpha}$ is large and positive. When $\boldsymbol{k}_{1}$ is very large and positive, $Y(t)$ will appear like a constant threshold of magnitude $k_{2} / k_{1}$ in a relatively short time. Typical trajectories will appear as in the general scheme depicted in Figure 1.

From the theory outlined above, the mean firing time, which we designate $F(x, y)$ for initial values $(\mathrm{X}(\mathrm{O}), \mathrm{Y}(\mathrm{O}))=(x, y)$, is the solution of

$$
\frac{\beta^{2}}{2} \frac{\partial^{2} F}{\partial x^{2}}+(\alpha-x) \frac{\partial F}{\partial x}+\left(k_{2}-k_{1} y\right) \frac{\partial F}{\partial y}=-1, \quad x<y,
$$

with $F(y, y)=0$. In general, this boundary-value problem will have to be solved numerically, though an asymptotic solution may be obtained for small $\boldsymbol{\beta}^{2}$ and/or at distances far away from the origin, i.e. as $x^{2}+y^{2} \rightarrow \infty$.

For a finite-difference solution, we first solve the problem for a finite rectangle $\mathrm{S}$ shown in Figure 3 with $\boldsymbol{F}(\boldsymbol{x}, y)=0$ on the boundary $\boldsymbol{\partial S}$ of $\mathrm{S}$. Care has to be taken that the corner $\mathrm{C}$ of the rectangle is above the line $\mathrm{y}=k_{2} / k_{1} \equiv k$ which is the asymptotic value of the threshold $Y(t)$ as $t \rightarrow \infty$. If $\mathrm{C}$ were not above this line, as would be the case if the asymptotic barrier value were $k^{\prime}$, any trajectory which started above $k$ ' could never reach the absorbing edge $\mathrm{y}=\boldsymbol{x}$. Such initial values would lead to infinite exit times and a solution on $S$ would not be possible.

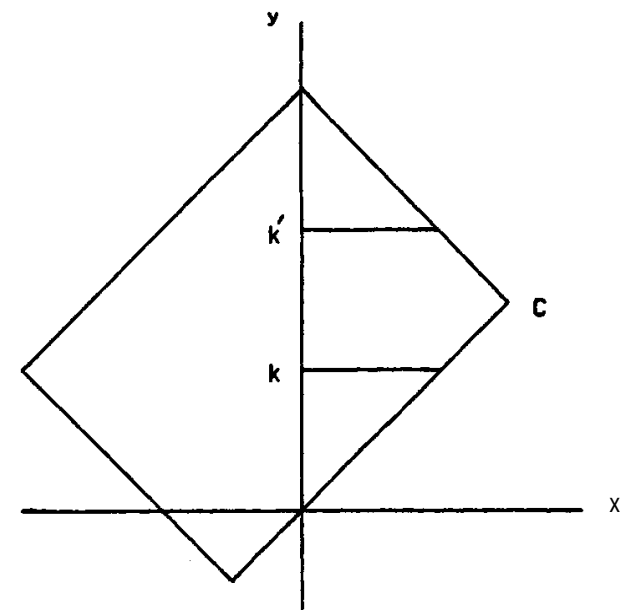

Figure 3. Illustrating the selection of the domain of the equation (4.4) in the neuron firing-time problem. 
We solved (4.4) numerically for various parameter values. For the neuronfiring problem we are mainly interested in the solution along $\mathrm{x}=0$ for various $\mathrm{y}$, representing thresholds with various initial values. The results are given in Table 1.

TABLE 1

Mean first-passage times of an Ornstein-UhIenbeck process, $X(t)$ to an exponentially decaying barrier, $\mathrm{Y}(\mathrm{t})$ with $X(O)=0, Y(0)=y$

\begin{tabular}{rc}
\hline A. $\boldsymbol{\alpha}=20, \boldsymbol{\beta}=10, \boldsymbol{k}_{\mathbf{1}}=\mathbf{1 ,} \boldsymbol{k}_{\mathbf{2}}=10$ \\
\hline $\boldsymbol{y}$ & $\boldsymbol{F}(\mathbf{0}, \boldsymbol{y}) \equiv \mathbf{M}_{\mathbf{1}} \mathbf{( 0 , y )}$ \\
\hline 8 & 0.479 \\
9 & 0.529 \\
10 & 0.577 \\
11 & 0.623 \\
12 & 0.666 \\
13 & 0.708 \\
14 & 0.747 \\
15 & 0.786 \\
\hline
\end{tabular}

\begin{tabular}{cc} 
B. $\alpha=20, \boldsymbol{\beta}=10, \boldsymbol{k}_{\mathbf{1}}=0.8, \boldsymbol{k}_{\mathbf{2}}=10$ \\
\hline $\boldsymbol{y}$ & $\boldsymbol{F}(\mathbf{0}, \boldsymbol{y}) \equiv \boldsymbol{M}_{\mathbf{1}}(\mathbf{0}, \boldsymbol{y})$ \\
\hline 8 & 0.534 \\
9 & 0.594 \\
10 & 0.652 \\
11 & 0.707 \\
12 & 0.760 \\
13 & 0.812 \\
14 & 0.862 \\
15 & 0.911
\end{tabular}

\begin{tabular}{rc}
\hline C. $\alpha=20, \beta=5, k_{1}=1, k_{2}=10$ \\
\hline$y$ & $F(0, y) \equiv M_{1}(0, y)$ \\
\hline 8 & 0.552 \\
9 & 0.604 \\
10 & 0.654 \\
11 & 0.701 \\
12 & 0.747 \\
13 & 0.790 \\
14 & 0.832 \\
15 & 0.872 \\
\hline
\end{tabular}

D. $\alpha=20, \beta=10, k_{1}=1, k_{2}=8$

\begin{tabular}{rc}
\hline$y$ & $\boldsymbol{F}(0, \boldsymbol{y}) \equiv \boldsymbol{M}_{\mathbf{1}}(\mathbf{0}, \boldsymbol{y})$ \\
\hline 6 & 0.326 \\
7 & 0.379 \\
8 & 0.428 \\
9 & 0.475 \\
10 & 0.520 \\
11 & 0.562 \\
12 & 0.603 \\
13 & 0.641
\end{tabular}

\begin{tabular}{cc}
\hline $\begin{array}{c}\text { E. } \boldsymbol{\alpha}=15, \boldsymbol{\beta}=10, \boldsymbol{k}_{\mathbf{1}}=1, \boldsymbol{k}_{\mathbf{2}}=10 \\
\left.\mathrm{~F}(0, \mathrm{y})=\boldsymbol{M}_{\mathbf{1}} \mathbf{( 0 ,} \mathrm{Y}\right)\end{array}$ \\
\hline 8 & 0.631 \\
9 & 0.699 \\
10 & 0.762 \\
11 & 0.821 \\
12 & 0.877 \\
13 & 0.929 \\
14 & 0.979 \\
15 & 1.026 \\
\hline
\end{tabular}


In all cases the results are such that a substantial increase in the size of $\mathrm{S}$ led to a less than 1 per cent change in the solution at the tabulated values. The solution must also be a monotonically increasing function of y on $\boldsymbol{x}=0$. The value of $F(0,10)$ in Case A of Table 1 was checked against a previously known value, since this represents the constant threshold solution $Y(t)=10, I \geqq 0$. It will be seen by examining the parameter values in Cases A-E that the qualitative dependence of the solution on the parameters is correct. Thus it is possible to obtain mean first-passage times of diffusion processes to many barriers in cases where these quantities were not previously known.

\section{Extensions}

The method outlined above for finding the moments of the time of first passage of a diffusion process to a moving barrier can be applied in the case of a Markov process $\mathrm{X}(\mathrm{t})$ with diffusion and jump components. Such a process will have a stochastic differential of the form

$$
d X=\alpha(X) d t+\beta(X) d W+_{I_{R}} \gamma(X, u) \nu(d t \times d u), \quad X(0)=x
$$

where $\nu()$ is a Poisson random measure (Gihman and Skorohod (1972)). The transition density $\mathrm{p}(\mathrm{s}, \mathrm{x} ; \mathrm{t}, \mathrm{y})$ of such a process satisfies a backward Kolmogorov equation

$$
-\frac{\partial p}{\partial s}=L_{x}[p]
$$

where

$$
L_{x}[p] \equiv \alpha(x) \frac{\partial p}{\partial x}+\frac{1}{2} \beta^{2}(x) \frac{\partial^{2} p}{\partial x^{2}}+\int_{R} p(s, x+\gamma(x, u) ; t, y) \Pi(d u)-\Lambda p,
$$

where $\Pi(\cdot)$ is the jump rate measure and $\mathrm{A}=\int \Pi(d u)$ is the total jump rate.

(i) Processes confined between two moving barriers. Suppose a Markov process $X(t)$ with stochastic differential given by (5.1) is initially between the two time-varying barriers $Y(t)$ and $Z(t)$, with $Y(O)>Z(O)$. Let $Y(t)$ and $Z(t)$ satisfy the differential equations

$$
\begin{array}{ll}
\frac{d Y}{d t}=f(Y), & Y(0)=y, \\
\frac{d Z}{d t}=g(Z), & Z(0)=z .
\end{array}
$$

We consider the random variable $T(x, y, z)$, the time at which $X(t)$ first hits either $Y(t)$ or $Z(t)$. If the moments $M_{n}(x, y, z)$ of $T$ exist, then $M_{n}, n=1,2$, satisfy the equations (Dynkin (1965)), 


$$
L_{x}\left[M_{n}\right]+f(y) \frac{\partial M_{n}}{\partial y}+g(z) \frac{\partial M_{n}}{\partial z}=-n M_{n-1},
$$

where the domain of the equations is the region $S=\{(x, y, z) \mid x<y, x>z\}$ and the boundary conditions are $M_{n}(x, y, z)=0,(x, y, z) \notin S$.

(ii) The density of the first-passage time. Consider a Markov process $X$, with stochastic differential (5.1), which is initially below a barrier $\mathrm{Y}(\mathrm{t})$, where $\mathrm{Y}(\mathrm{t})$ satisfies (5.4). Let the distribution of the time $T(x, y)$ at which $\mathrm{X}$ first reaches or exceeds $\mathrm{Y}$ be

$$
G(t, x, y)=\operatorname{Pr}[T(x, y) \leqq t] .
$$

From the theory of exit times for Markov processes, G will satisfy the equation

$$
\frac{\partial G}{\partial t}=L_{x}[G]+f(y) \frac{\partial G}{\partial y}, \quad t>0, x<y,
$$

with boundary conditions

$$
\begin{gathered}
G(t, x, y)=1, \quad t \geqq 0, \quad x \geqq y, \\
G(0, x, y)=0, \quad x<y .
\end{gathered}
$$

The density $g(t, x, y)$ of $\mathrm{G}$, if it exists, will satisfy the equation

$$
\frac{\partial g}{\partial t}=L_{x}[g]+f(y) \frac{\partial g}{\partial y}, \quad t>0, \quad x<y,
$$

with boundarv conditions

$$
\begin{gathered}
g(t, x, y)=\delta(t), \quad x \geqq y, \\
g(0, x, y)=0, \quad x<y .
\end{gathered}
$$

Similarly, equations for the distribution function and density of the time at which a Markov process $X(t)$, with stochastic differential (5.1), leaves the region bounded by $Y(t)$ and $Z(t)$ satisfying (5.4) and (5.5), may be obtained.

For example, the density of first-passage-time density of Problem (iii) in Section 3 satisfies

$$
\frac{\partial g}{\partial t}=\frac{\sigma^{2}}{2} x^{2} \frac{\partial^{2} g}{\partial x^{2}}+\left(\mu+\frac{\sigma^{2}}{2}\right) x \frac{\partial g}{\partial x}+k y \frac{\partial g}{\partial y} \quad(t>0, x<y) .
$$

The solution of this equation satisfying (5.12) and (5.13) is

$$
g(t, x, y)=\frac{\ln /\left(y / y_{-}\right),}{\sqrt{2 \pi \sigma^{2} t^{3}}} \exp \left[\frac{-(\ln y / x)}{2 \sigma^{2} t}-\mu t^{2}\right), \quad(t>0)
$$

as can be seen by transforming the process $\mathrm{X}$ to a Wiener process and the barrier to a constant one. 
(iii) Barriers satisfying higher -order differential equations. If the barrier $Y(t)$ satisfies a differential equation of order greater than 1 , the moments and density of the time at which the random process $X(t)$ first hits $Y(t)$ may sometimes be found if the equation for $Y(t)$ can be written as an autonomous system of first-order equations. To illustrate, suppose $\mathrm{X}(\mathrm{t})$ has a stochastic differential (5.1) and the barrier satisfies the deterministic second-order equation

$$
\frac{d^{2} Y}{d t^{2}}-f(Y) \frac{d Y}{d t}-g(Y)=0, \quad Y(0)=y, \quad \dot{Y}(0)=z
$$

The second-order ODE for Y may be written as a first-order system

$$
\frac{d Y}{d t}=Z, \quad \frac{d Z}{d t}=f(Y) Z+g(Y)
$$

Let $T(x, y, z)$ be the time at which $X(t)$ first hits $Y(\mathrm{t})$ when $[X(0), Y(0), Z(0)]=(x, y, z)$. Then the moments $M_{n}(x, y, z)$ of $T$ satisfy the equations

$$
L_{x}\left[M_{n}\right]+z \frac{\partial M_{n}}{\partial y}+[f(y) z+g(y)] \frac{\partial M_{n}}{\partial z}=-n M_{n-1},
$$

in the half-space $x<y$, with boundary conditions $M_{n}(x, y, z)=0, x \geqq y$. Similarly, the distribution function $G$ of $T$ satisfies

$$
\frac{\partial G}{\partial t}=L_{x}[G]+z \frac{\partial G}{\partial y}+[f(y) z+g(y)] \frac{\partial G}{\partial z}, \quad(t>0, x<y),
$$

with boundary conditions

$$
\begin{gathered}
G(t, x, y, z)=1, \quad(t \geqq 0, x \geqq y), \\
G(0, x, y, z)=0, \quad(x<y) .
\end{gathered}
$$

(iv) First-passage time of a Wiener process with drift to a sinusoidal barrier. For a specific application of the method for barriers satisfying a higher-order ODE, we consider the Weiner process with drift

$$
d X=\mu d t+\sigma d W, \quad X(O)=x,
$$

and let $T(x, y, z)$ be the time at which $X(t)$ first reaches the barrier $Y(t)$ satisfying

$$
\frac{d^{2} Y}{d t^{2}}+\omega^{2}(Y-k)=0, \quad Y(0)=y, \quad \dot{Y}(0)=z
$$

Thus the barrier is the sinusoidal function of time

$$
Y(t)=(y-k) \cos (\omega t)+\frac{z}{\omega} \sin (\omega t)+k
$$


with a 'mean value' of $k$ and a period $2 \pi / \omega$. Then, from (5.18) the expected time $M_{1}$ at which $X(t)$ first hits $Y(t)$ satisfies the equation

$$
\frac{1}{2} \sigma^{2} \frac{\partial^{2} M_{1}}{\partial x^{2}}+\mu \frac{\partial M_{1}}{\partial x}+z \frac{\partial M_{1}}{\partial y}+\omega^{2}(y-k) \frac{\partial M_{1}}{\partial z}=-1, \quad(x<y),
$$

where it is assumed that passage of $X(t)$ to $Y(t)$ is certain in a finite time. An asymptotic solution of (5.25) with boundary condition $M_{1}(x, y, z)=0$ in terms of elementary functions is possible when $\boldsymbol{\sigma}^{2}$ is sufficiently small. For the more general situation, a numerical solution may be obtained as in Section 4 .

\section{Acknowledgements}

The authors are grateful to Professor Y. S. Wong of McGill University and $\mathrm{Mr}$ Mark Parankowski of the University of British Columbia for their help with the computational aspects of this paper.

\section{References}

BLAKE, I. F. AND LiNDSEY, W. C. (1973) Level crossing problems for random processesIEEE Trans. Information Theory 19, 295-315.

Cox, D. R. And Miller, H. D. (1965) The Theory of Stochastic Processes. Wiley, New York.

Crow, 1. F. AND KIMURA, M. (1970) An Introduction to Population Genetics Theory. Harper and Row, New York.

DARLING, D. A. AND SIEGERT, A. J. F. (1953) The first passage problem for a continuous Markov process. Ann. Math. Statist. 24, 624-639.

DURBIN, J. (1971) Boundary-crossing probabilities for the Brownian motion and Poisson processes and techniques for computing the power of the Kolmogorov-Smirnov test.J.Appl. Prob. 8, $431-453$.

DYNKIN, E. B. (1965) Markov Processes, I and II. Springer-Verlag, Berlin.

EWENS, W. J. (1979) Mathematical Population Genetics. Springer-Verlag, Berlin.

FEREBEE, B. (1982) The tangent approximation to one-sided Brownian exit densities. Z. Wahrscheinlichkeitsth 61, 309-326. Berlin.

GIHMAN, I. I. AND SкоRоноD, A. V. (1972)Stochastic Differential Equations. Springer-Verlag,

GLUSS, B. (1967) A model for neuron firing with exponential decay of potential resulting in diffusion equations for probability density.BuIl. Math. Biophys. 29, 233-243.

HOLDEN, A. V. (1976) Models of the Stochastic Activity of Neurones. Springer-Verlag, Berlin.

Jaswinski, A. H. (1970) Stochastic Processes and Filtering Theory. Academic Press, New York.

Roy, B. K. AND SMith, D. R. (1969) Analysis of exponential decay model of the neuron showing frequency threshold effects. Bull. Math. Biophys. 31, 341-357.

SteIN, R. B. (1965) A theoretical analysis of neuronal variability. Biophys. J. 5, 173-194.

Tuckweli, H. C. (1974) A study of some diffusion models of population growthheoret. Popn Biol. 5, 345-357.

TUCKWELL, H. C. (1976) The effects of random selection on gene frequency.Math. Biosci. 30, 113-126.

TUCKWELl, H. C., Wan, F. Y. M. AND WONG, Y. S. (1984) The interspike interval of a cable model neuron with white noise input. Biol. Cybernet. 49, 155-167. 
Wan, F. Y. M., wong, Y. S. ANd TuCKwell, H. C. (1983) Firing time of spatially distributed neurons and vector valued diffusion processes. I.A.M.S. Technical Report.

WeIss, R. D. (1964) A model for firing patterns of auditory nerve fibers. MIT. - Research Lab. of Electronics, Tech. Report \#418.

Whbur, W. J. and RINZEL, J. (1983) A theoretical basis for large coefficient of variation and bimodality in neuronal interspike interval distributions. J. Theoret. Biol. 105, 345-368. 